\title{
Characterisation and Categorisation Strategies for Anisotropic Gold Nanoparticles for Applications in Biology
}

\author{
Jennifer Cookman ${ }^{1 *}$, João. M. de Araujo² and Kenneth A. Dawson ${ }^{3}$ \\ 1. Bernal Institute, University of Limerick, Castletroy, Limerick, Ireland. \\ 2. Departamento de Físíca Teóríca e Experimental, Universidade Federal do Río Grande do Norte, Natal, \\ RN, Brazil. \\ 3. Centre for BioNano Interactions, University College Dublin, Belfield, Dublin, Ireland. \\ * Coresponding author: Jennifer.Cookman@ul.ie
}

This body of work focusses on understanding the morphology of nanoparticles (NP) by obtaining 3D models of single NPs from Transmission Electron Microscope (TEM) tomography. The wireframe mesh obtained from the 3D models opens avenues to compute parameters of the NPs' geometry such as its surface area and volume. The wireframe can also be used to obtain a shape specific signature (denoted D2) to characterise its shape with a view to standardise nomenclature of anisotropic NP.

It is known that as nanomaterials are examined in a biological context, their interaction with the biological environment must be acknowledged prior to concluding any independent influences the nanomaterials shape and/or material may have. For non-spherical and anisotropic nanomaterials, this becomes more difficult due to the local bio-nano interactions at the biological interface for various morphologies.

It has been rational to call complex nanomaterial shapes after recognised objects such as stars [1], urchins [2], disco-balls [3] and even cauliflower [4]. This method of nomenclature has caused many procedures being claimed individual to create essentially the same geometry of the same material. The need to create a standardised method of recalling nanoparticles and their significant properties is becoming more paramount especially when we consider IUPAC nomenclature for molecules has existed in its official form since 1919 [5].

To characterise non-spherical shapes more thoroughly, the described method presented uses electron tomography to result in 3D models of individual nanoparticles similar to single particle. 121 micrographs were obtained via TEM tomography with $1^{\circ}$ increment from $-60^{\circ}$ to $+60^{\circ}$. The data obtained was processed using IMOD software suite to gain a $3 \mathrm{D}$ volume with the nanoparticle object contained. Segmentation software (commonly used for CT scans) is then used to produce a 3D model of the nanoparticle from which the wireframe mesh can be obtained to compute geometric parameters. To obtain the geometric parameters i.e. surface area and volume in-house built software was used and finally the D2 signatures were generated using a previously reported method for computer graphics [6].

The 3D models are then used to build a database of shaped nanoparticles from which individual morphological signatures can be calculated, termed D2 signatures. The D2 signatures of the individual nanoparticle 3D models are then used to categorise shapes by comparing to the other 3D models of shaped nanoparticles and also the 3D models of theoretical shapes. The categorisation of shaped nanomaterials in this context furthers their full characterisation prior to their utility in bio-nano applications [7]. 


\section{References:}

[1] Yuan, H. et al., Nanotechnology 23 (2012), p. 075102.

[2] Su, Y. H. et al., Nanoscale 2 (2010), p. 2639.

[3] Chhour, P. et al., ACS nano 8 (2014), p. 9143.

[4] Ren, H.-X. et al., Journal of Colloid and Interface Science 334 (2009), p. 103.

[5] Secretariat, I. The International Union of Pure and Applied Chemistry (IUPAC). (accessed 2/16/17)

[6] Osada, R. et al., ACM Transactions on Graphics (TOG) 21 (2002), p. 807.

[7] The authors acknowledge funding from EU FP7 Future Nano Needs and discussions from members of the CBNI research group

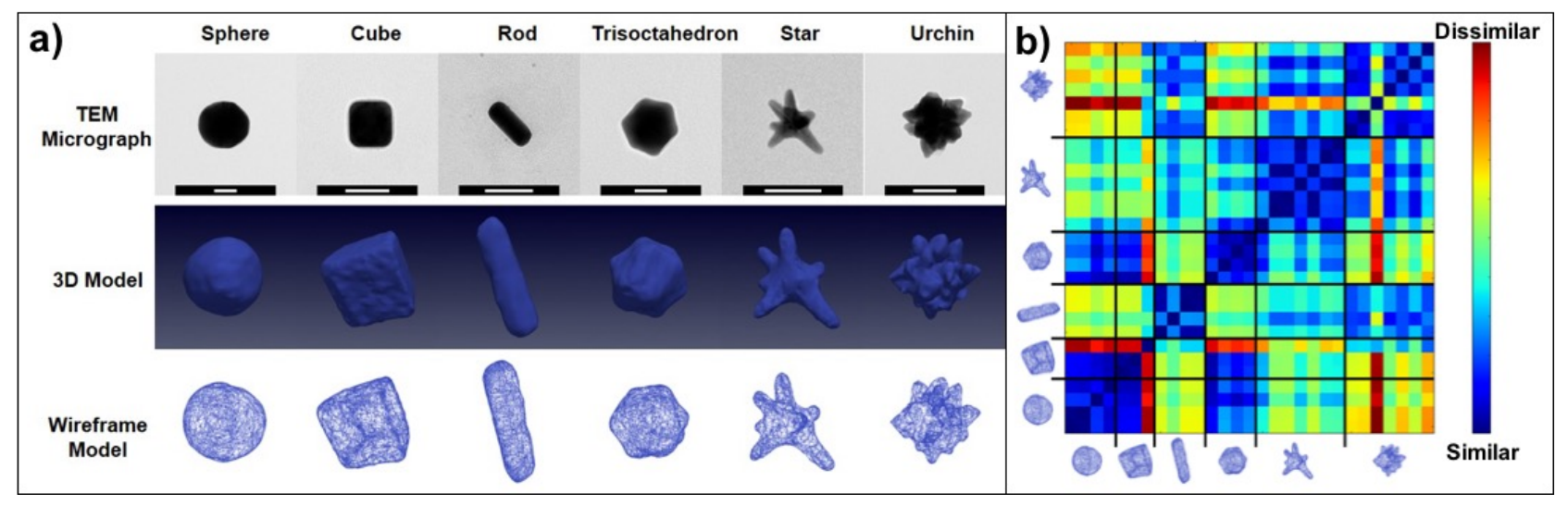

Figure 1. a) For each of the six shaped nanoparticles; sphere, cube, rod, trisoctahedron, star and urchin, a 3D model was created from the tomogram data. The 3D model is composed of a wireframe mesh model, which is further utilised in the D2 distribution and finally in b) the dissimilarity matrix as a comparison method and categorisation strategy for shaped singe nanoparticles. Scale bar: $50 \mathrm{~nm}$. 